\title{
Polymerase chain reaction and DNA sequencing
}

\author{
Ricardo Escalante
}

Instituto de Investigaciones Biomédicas del CSIC-UAM

C./ Arturo Duperier, 4. 28029 Madrid.

Polymerase chain reaction (PCR) is one of the most powerful tools available for studying nucleic acids. To understand the power of this technique, we need to imagine how difficult it would be to find a specific sequence of DNA of 500 base pairs in the entire human genome, of approximately $3,300,000,000$ base pairs. Not only is PCR able to carry out this task but it can also present the isolated DNA in sufficient amounts for it to be used in other molecular biology techniques, such as cloning and sequencing. Sequencing makes it possible to identify a specific mutation in a base pair that might be essential for diagnosis.

Biological material is often available in limited amount. However, PCR is sensitive enough to successfully amplify even extremely small samples.

\section{BASIC CONCEPTS OF PCR}

DNA molecules are formed by two complementary chains. The specific sequence of the bases (A, C, $T, G)$ contains the genetic information for each protein. This sequence is therefore specific for each gene. In the absence of one of the two complementary chains, polymerases can copy the other chain to complete the original double-straded DNA molecule. Polymerases use deoxynucleotides (dNTPs) as substrates. The complementary chain will be constructed with the pairs $\mathrm{A}-\mathrm{T}$ and $\mathrm{G}-\mathrm{C}$.

In the PCR, two specific primers (small fragments of single-stranded DNA) must match the sequence of each complementary chain at the extremes of the fragment that is to be amplified. In the first step (melting), the sample is heated to induce the melting of the chains. In the second step (annealing), the temperature is lowered to allow the primers to interact and hybridize to their specific sequences in the template DNA. In the third step (extension), the temperature is raised again to reach the optimum so that the polymerase synthesizes the complementary chains. This sequence of events or cycles (melting-annealing-extension) is repeated 30 to 40 times, and the amount of DNA will double with each cycle. This means that PCR is an exponential process, generating a large number of molecules. Fi gure 1 shows the PCR mechanism.

Several variables affect both the yield and the specificity of a PCR reaction: temperature and length of the steps, number of cycles, concentration of the different components (DNA, template, polymerase, primers, dNTPs), buffer composition, and quality of the template. In most cases, the standard protocols give satisfactory results, but sometimes it is necessary to find specific conditions to avoid lack of specificity or low yield. In these cases, the slight variation of any $\circ$

these variables might be critical. Detailed information can be obtained in several technical books that are cited in the bibliography. Figure 1 shows a PCR reaction over the first rounds. After 30 cycles the amount of DNA is huge.

REVERSE TRANSCRIPTASE PCR

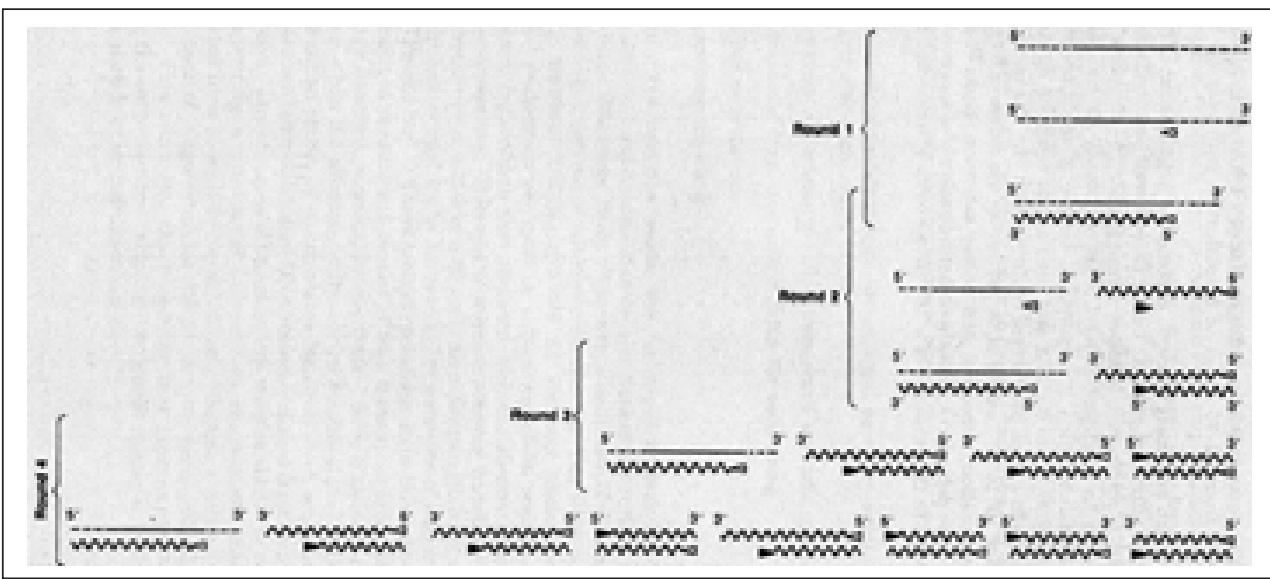

Fig. 1. First rounds of a PCR reaction. 


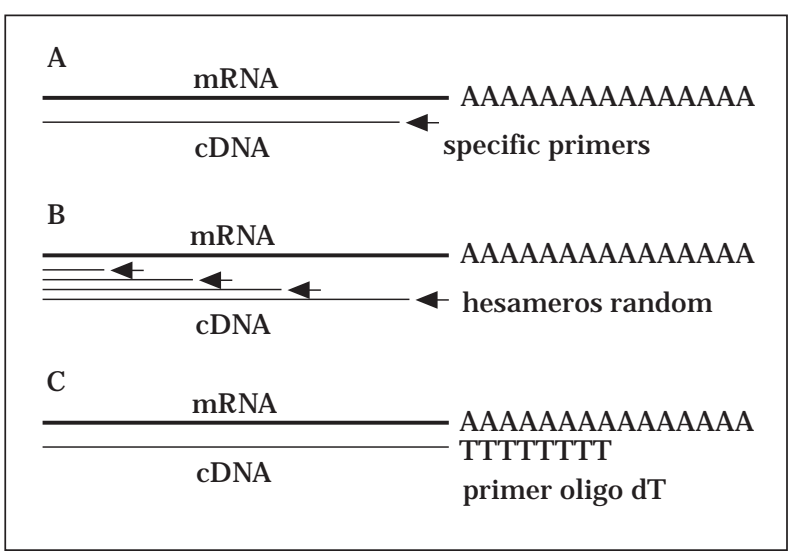

Fig. 2. Types of primers used for reverse transcripton reactions.

Reverse transcriptase PCR (RT-PCR) is a PCR technique that allows amplification from RNA molecules. Eukaryotic genes are interrupted by non-coding sequences named introns that are eliminated in the maturation of the RNA by splicing. The presence of introns sometimes makes it impossible to amplify a specific fragment due to its length. By using RNA as a starting point for PCR, we avoid the presence of introns in the sequence we desire to amplify. Since DNA polymerases can only use DNA as a template, another enzyme has to be used: reverse transcriptase. This enzyme is able to use RNA as a template to synthesize a complementary DNA molecule. This DNA template in turn can be used as a template in a standard PCR reaction. For the reverse transcription step, several kinds of primers can be used: specific primers of the sequence that will later be amplified; small random primers that will prime nonspecifically to any RNA molecules; or a poli-dT primer that will recognize the poly-A tail. These possibilities are shown in figure 2 .

\section{QUANTITATIVE PCR: REAL TIME PCR OR TAQMAN}

In standard conditions, the amount of a PCR-amplified fragment of DNA is not proportional to the original amount of specific template in the sample. I

other words, PCR is not quantitative. However, under controlled conditions and with the correct controls, we may be able to make it quantitative. This is very important since PCR, due to its sensitivity, may be the only way to detect a specific DNA in a very small sample. However, it may be necessary for us not only to detect that DNA but also to determine the amount present in the original sample. Several protocols for quantitative

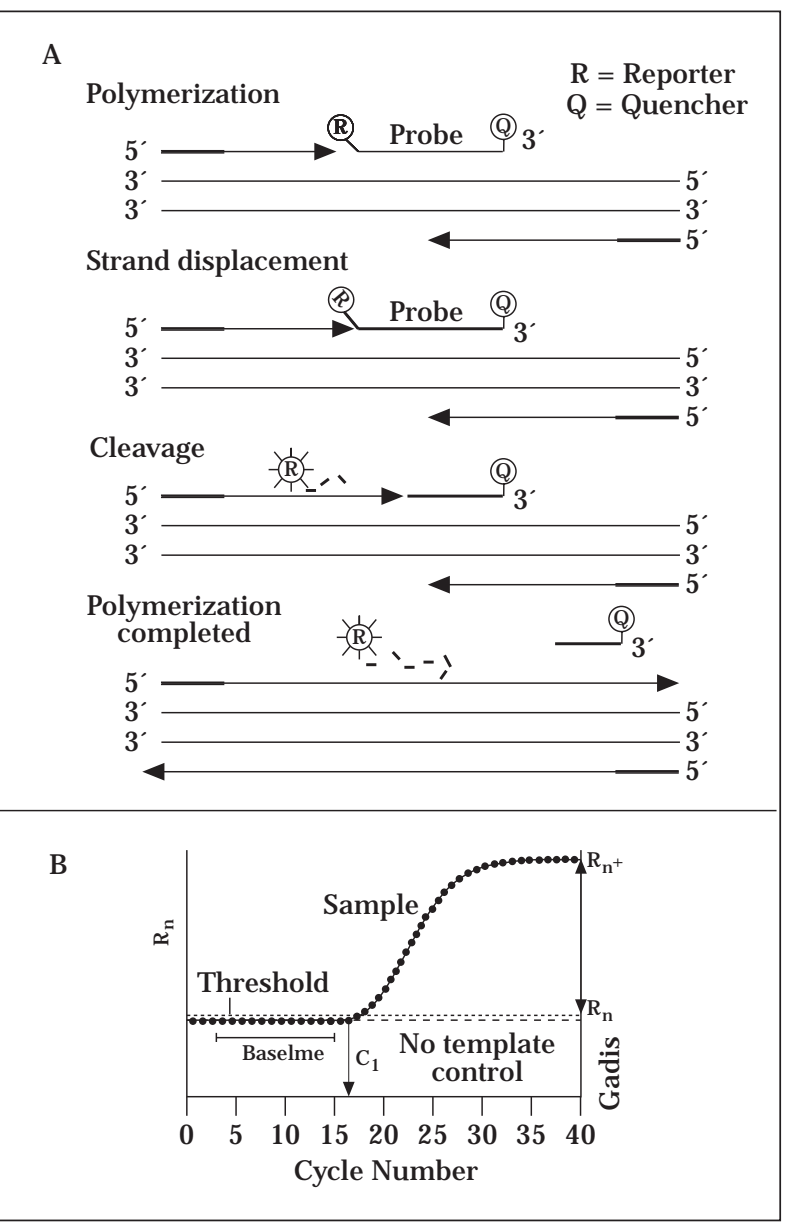

Fig. 3. Quantitative. PCR: real time PCR or Taqman. A: principle of the technique. B: graphic of CT calculation.

PCR are available, but in the last few years a very powerful technique called Taqman is being used for that purpose.

The principle of this technique (fig. $3 \mathrm{~A}$ ) is based on the presence in the PCR reaction of a complementary oligonucleotide in the middle of the sequence. This oligo (also called a probe) contains a reporter in one end (which provides fluorescence) and a quencher in the other. When this oligo is intact, the proximity of the reporter to the quencher inhibits the fluorescence of the reported by squelching.

During the extension step in the PCR reaction, the Taq-polymerase will degrade the probe separating the reporter and the quencher and bringing both into solution. The reporter fluorescence is no longer inhibited and is now detectable by the machine. Every cycle of the PCR is monitored and the fluorescence quantified and plotted. In the example of figure $3 \mathrm{~B}$, we can see how an exponential region is reached at approximately cycle 17. This number (ct) is related to the amount of the specific 


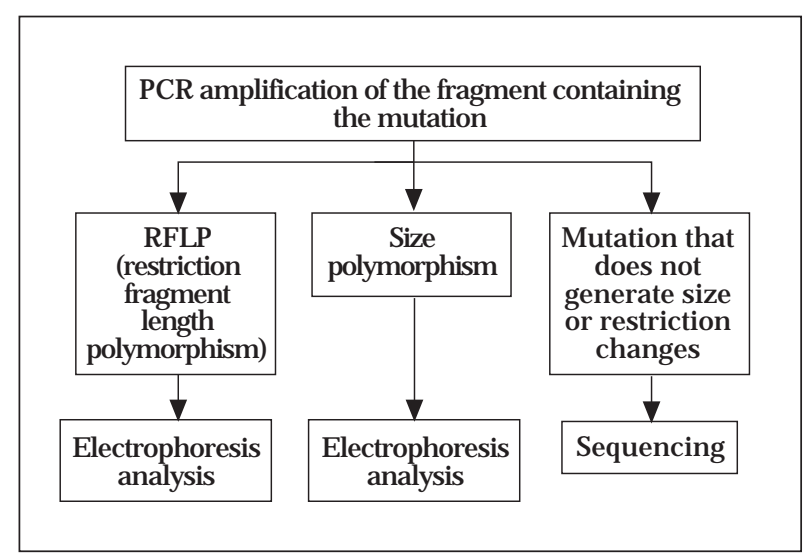

Fig. 4. Scheme shows some of the type of analysis performed after PCR amplification.

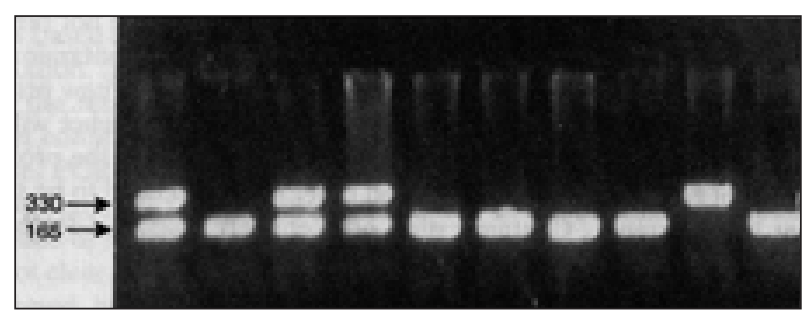

Fig. 5. Gel of PCR-generated fragments cut with a polymorphic restriction enzyme.

mRNA in the original sample. A simultaneous experiment with different known quantities of another control RNA will allow us to extrapolate the Ct value into a quantative value.

\section{PCR AND DIAGNOSIS}

PCR represents a real breakthrough in the field of genetic diagnosis. Before PCR, a typical assay to detect a polymorphism required the isolation of large quantities of DNA. This DNA was cut with restriction enzymes and analyzed by radioactive hybridization in a Southern blot. No less than 5 days would be necessary. With the advent of PCR, the same result can be obtained in few hours. Figure 4 shows the most frequent type of analysis performed over the amplified fragment to detect specific polymorphisms. In all these cases, we must know the nature of the mutation wer are looking for. If this mutation generates changes in the recognition site of a given restriction enzyme or changes in the size of the amplified product, the analysis is simple and fast. If these changes are not generated, it is neccessary to sequence the amplified product. Figure 5 shows a gel in which PCR-generated fragments were cut with a polymorphic restriction enzyme. The band of the PCR is approximately 300 base pairs long. After digestion, the size of the band will remain the same if the restriction enzyme site is not present, or it will divide into two fragments of approximately $150 \mathrm{pb}$ that run as an apparent single band. In the example of figure 5, each lane corresponds to one individual. Both homozygosis (the presence of one band) and heterozygosis (the presence of two bands) can easily be detected.

\section{PCR AND MUTATIONAL ANALYSIS}

The use of PCR for mutational analysis is very common, and several techniques are available.

Single-stranded conformation polymorphism (SSCP) is a very sensitive technique based on the different conformations that single-stranded DNA can adopt due to a mutation. This different conformation leads to different migration properties under electrophoresis. The samples are compared with wild type to detect abnormal migration of the bands. Sequencing is necessary to confirm the possible mutation.

Heteroduplex analysis is based on the formation of duplex chains between wild type and mutants. These hybrid DNA molecules will run differently if they contain mismatches due to the mutation. Sequencing is necessary to ascertain the nature of the mutation.

\section{DNA SEQUENCING}

It is evident how important it is to know the sequence of the DNA coding for proteins. Determination

these sequences may lead to the discovery of

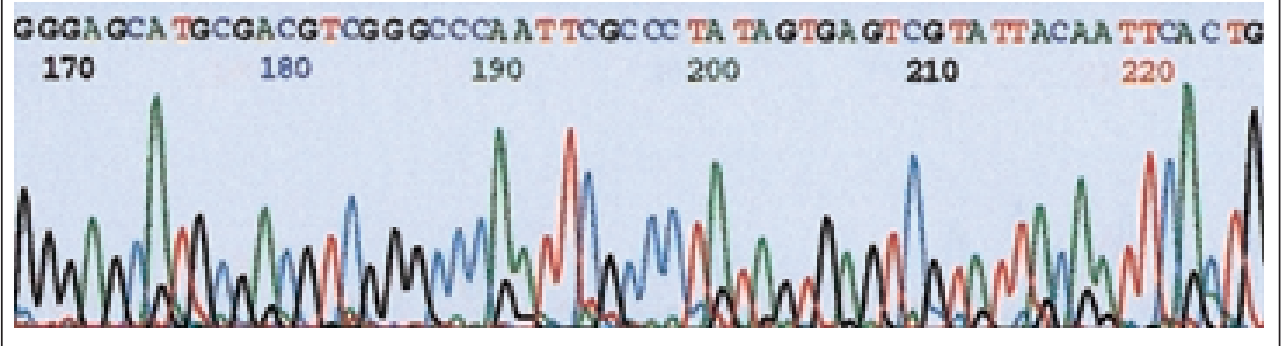

Fig. 6. Sequencing image based on the sanger method as provided by the sequencing machine. 
specific mutations that might be essential for diagnosis purposes. In basic science, sequencing is the base for the discovery of new genes. The genome project is devoted to determining the entire sequence of the human DNA. This task has been completed in other simpler organism like bacte- ria, yeast or Drosophila, and it has been shown to be a big step forward in the understanding of the function of all genes in a living organism.

Sequencing tools began to be developed in the 70s. In fact the methods by Sanger and Gilbert published in 1977 were awarded the Nobel Prize. Since them, optimization of those methods (mainly Sanger's) has allowed sequencing to be an easy and reliable technique. Figure 6 shows a typical sequencing result based on the Sanger method with the aid of PCR.

Recomended references

PCR: a practical approach. En: McPherson MJ, Quirke P, Taylor GR, eds. General introduction for those who are not very familiar with PCR. IR Press, 1991.

PCR primer: a laboratory manual. En: Dieffebach CW, Dveksler GS. Detailed protocol book for the laboratory. Cold Spring Harbor Laboratory Press, 1995.

Baumforth KRN, Nelson PN, Digby JE, O'Neil JD, Murray PG. Demystified.. the polymerase chain reaction. Basic review. J Clin Pathol: Mol Pathol 1999; 52.

PCR applications. Protocols for functional genomics. En: Innis MA, Gelfand DH, Sninsky JJ, eds. An update book that includes the Real Time PCR technique. Academic Press, 1999. 